\title{
CONSTRAINTS ON THE BIASING OF DENSITY FLUCTUATIONS
}

\author{
ALEXANDER S. SZALAY \\ Dept. of Physics and Astronomy, The Johns Hopkins University \\ and \\ Dept. of Atomic Physics, Eötvös University, Budapest
}

\begin{abstract}
A general nonlinear function $G(X)$ describing the biasing of primordial Gaussian density fluctuations is considered. Arbitrary N-point correlations of the biased field are calculated in the form of a series expansion in terms of the correlations of the Gaussian field. The observed scaling of the three point correlations in the galaxy distribution is satisfied, but the scaling coefficient $Q$ has a nontrivial value $Q=J_{2} / J_{1}^{2}$, where $J_{k}$ is the $k$-th term in the Hermite expansion of $G(X)$. The three point function is always accompanied by a cubic term $Q^{3} \xi_{1} \xi_{2} \xi_{3}$, independent of the functional form of the biasing. Its absence in the cluster 3-point correlations may be observable, in which case it rules out biasing as the major amplification mechanism of galaxy and cluster correlations.
\end{abstract}

\section{INTRODUCTION}

If galaxies formed by the nonlinear biasing of a Gaussian density fluctuation field, the correlation of galaxies is expected to exceed the correlations of the original fluctuations by a large factor (Kaiser 1984). Consider the density fluctuation field $\delta(\vec{x})=[\rho(\vec{x})-\langle\rho\rangle] /\langle\rho\rangle$, a Gaussian, with dispersion $\sigma^{2}=\left\langle|\delta|^{2}\right\rangle$. To simplify the notation we will use the normalized random field $y=\delta / \sigma$. The correlation function of the $y$ field is $w_{12}(r)=\left\langle y_{1} y_{2}\right\rangle$, where $\left|\vec{x}_{1}-\vec{x}_{2}\right|=r$. If we "clip" the normalized $y$ field at a level $\nu$, i.e. we set the value of the field to 1 , if $y>\nu$ and to 0 otherwise, and associate galaxies with the regions above this level, the two point correlations become with a good approximation (Politzer and Wise 1984, Jensen and Szalay 1986, hereafter PW and JS),

$$
1+\xi_{12}=e^{\nu^{2} w_{12}} .
$$

On the other hand, if we do a soft clipping by using an exponential (Kaiser and Davis 1985), creating a luminosity density proportional to $G(y) \propto \exp (\nu y)$, the correlation function is the same. The $\mathrm{PW}$ result is only an approximation, obtained from the linear expansion of the exponent in the bivariate Gaussian distribution. The exact result for sharp clipping was obtained by JS, using a series expansion. 
Fry (1986) considered a power law biasing, $G(y)=A(1+y)^{\alpha}$, as an example of generating non-Gaussian distribution. Another form of biasing, $G(y)=\exp (\alpha y-$ $\left.\beta y^{2} / 2\right)$ was used to include the modulating effects of a large scale background field on the density of galaxies, associated with the peaks of the overdensity filtered on galactic mass scales (Eq.(5.6) and (6.36), Bardeen et al. 1986, hereafter BBKS), with similar correlation functions.

How specific are these results? Are they simply due to the nonlinearity of the clipping, or do they depend on the detailed shape of the threshold function? If so, what are the dominant features, and how free is one to choose a threshold function without seriously changing the final result? We adress these questions below, discuss a general formalism and calculate the various $\mathrm{N}$-point correlation functions of the biased random field to arbitrary accuracy, using a method similar to the one used by JS. Such a treatment was also discussed from a different point of view by Taquu (1977).

\section{THRESHOLD FUNCTIONS}

We consider a general nonlinear threshold function $G(y)$, describing how the local luminosity density of galaxies depends on the Gaussian fluctuations of the mass, representing the effect of biasing. $G(y)$ should have non-negative values only. The 'clipping' would correspond to a step function, $G(y)=A \Theta(y-\nu)$. Hereafter we will denote the two point correlations of the galaxies with $\xi_{12}$, while $w_{12}=\left\langle y_{1} y_{2}\right\rangle$ is the two point correlation of the mass. $G(y)$ is normalized to unity :

$$
\langle G\rangle=\frac{1}{\sqrt{2 \pi}} \int_{-\infty}^{\infty} G(y) e^{-y^{2} / 2} d y=1
$$

One can expand the $G(y)$ function in terms of Hermite polynomials defined by $H_{n}(x)=e^{x^{2} / 2}\left(-\frac{d}{d x}\right)^{n} e^{-x^{2} / 2}$, normalized to $\left\langle H_{m}(x) H_{n}(x)\right\rangle=\delta_{m n} m !$.

$$
G(y)=\sum_{k=0}^{\infty} \frac{J_{k}}{k !} H_{k}(y)
$$

where

$$
J_{k}=\frac{1}{\sqrt{2 \pi}} \int_{-\infty}^{\infty} d y e^{-y^{2} / 2} G(y) H_{k}(y)=\left\langle G(y) H_{k}(y)\right\rangle .
$$

After simple calculations, using the orthogonality of the Hermite polynomials, we obtain the two point correlation

$$
\left\langle G\left(X_{1}\right) G\left(X_{2}\right)\right\rangle=1+\xi_{12}=\sum_{k=0}^{\infty} \frac{J_{k}^{2}}{k !} w_{12}^{k}
$$

where now $\langle\ldots\rangle$ is an integral over the bivariate Gaussian distribution function $f\left(X_{1}, X_{2}\right)$ with the correlation $w_{12}$. Therefore in the linear regime the leading term becomes

$$
\xi_{12} \approx J_{1}^{2} w_{12}
$$


In order to compare with previous results we calculate the $J_{k}$ coefficients for the case of sharp clipping:

$$
G(X)=A \Theta(X-\nu) \quad ; \quad J_{k}=\frac{\nu H_{k-1}(\nu)}{\nu \sqrt{\pi / 2} \exp \left(\nu^{2} / 2\right) \operatorname{erfc}(\nu / \sqrt{2})} \rightarrow \nu^{k}
$$

in agreement with the results obtained by JS. The value of $A$ is determined by the normalization condition Eq.(2). Taking the exponential (soft) clipping instead, characterized by the threshold function

$$
G(X)=A e^{\nu X} \quad ; \quad J_{k}=\nu^{k}
$$

the coefficients become particularly simple. Since all moments in the two cases are asymptotically equal, any results using the soft instead of the sharp clipping should be very similar, as they indeed are. We retrieve the result of PW and JS

$$
\left\langle G\left(X_{1}\right) G\left(X_{2}\right)\right\rangle=1+\xi_{12}=\sum_{k=0}^{\infty} \frac{\nu^{2 k} w_{12}^{k}}{k !}=e^{\nu^{2} w_{12}}
$$

One can easily generalize this result to more complicated cases, such as higher order correlation functions, or if we have more complicated constraints, density peaks (BBKS). In the general case we will consider a $p$-variate Gaussian, with a correlation matrix $M$. Each of the normal Gaussian variables $X_{i}$ is distorted by a different nonlinear function $G_{i}\left(X_{i}\right)$, with the $k$-th Hermite coefficients denoted by $J_{i}(k)$. We would like to calculate the expectation value $\left\langle G_{1}\left(X_{1}\right) \ldots G_{p}\left(X_{p}\right)\right\rangle$. By choosing appropriate $G_{i}$ functions, all moments of the $G$ 's can be expressed in this form. The joint probability distribution function is determined by the correlation matrix M, and can be expanded in terms of Hermite polynomials (Taquu 1977).

$$
\begin{aligned}
& f\left(X_{1}, \ldots, X_{p}\right)=\frac{1}{\sqrt{(2 \pi)^{p}|\mathbf{M}|}} \exp \left(-\frac{1}{2} \mathbf{X M}^{-1} \mathbf{X}\right)= \\
& \frac{1}{(2 \pi)^{p / 2}} \exp \left(-\frac{1}{2} \mathrm{X}^{2}\right) \sum_{n=0}^{\infty} \frac{1}{2^{n} n !} \sum_{k(n)} H_{k_{1}}\left(X_{1}\right) \ldots H_{k_{p}}\left(X_{p}\right) \sum_{i, j} w_{i_{1} j_{1}} \ldots w_{i_{n} j_{n}}
\end{aligned}
$$

Here $\sum_{k(n)}$ means summing over all $k_{i}$ satisfying $0 \leq k_{1}, \ldots, k_{p} \leq n$, and $k_{1}+$ $\ldots+k_{p}=2 n$, and $\sum_{i, j}$ is a sum over all pairs of $i_{k} \neq j_{k}$, which satisfy $1 \leq$ $i_{1}, j_{1}, \ldots i_{n}, j_{n} \leq p$, where there are $k_{1}$ indices 1 , and $k_{p}$ indices $p$.

Using the orthogonality of the Hermite-polynomials, one can then express the expectation value

$$
\begin{aligned}
\left\langle G_{1}\left(X_{1}\right) \ldots G_{p}\left(X_{p}\right)\right\rangle= & \int d X_{1} G_{1}\left(X_{1}\right) \ldots \int d X_{p} G_{p}\left(X_{p}\right) f\left(X_{1}, \ldots, X_{p}\right)= \\
& \sum_{n=0}^{\infty} \frac{1}{2^{n} n !} \sum_{k(n)} J_{1}\left(k_{1}\right) \ldots J_{p}\left(k_{p}\right) \sum_{i, j} w_{i_{1} j_{1}} \ldots w_{i_{n} j_{n}}
\end{aligned}
$$

This expression can be used to derive any of the N-point correlation functions of the biased density field to arbitrary accuracy. Potential applications include much more complicated cases than the ones discussed here, such as cross correlation of different objects selected by different $G_{i}$ functions. In the following, we will apply this to the special case of the three point correlations, and derive some general constraints. 


\section{THREE POINT CORRELATIONS}

Using Eq.(11) to express the 3 point correlations, we expand to third order in $w$, writing

$$
\begin{aligned}
\left\langle G_{1} G_{2} G_{3}\right\rangle & =1+\xi_{12}+\xi_{23}+\xi_{31}+\zeta_{123}=1+J_{1}^{2}\left(w_{12}+w_{23}+w_{31}\right)+ \\
& \frac{J_{2}^{2}}{2}\left(w_{12}^{2}+w_{23}^{2}+w_{31}^{2}\right)+J_{1}^{2} J_{2}\left(w_{12} w_{23}+w_{23} w_{31}+w_{31} w_{12}\right)+ \\
& \frac{J_{3}^{2}}{6}\left(w_{12}^{3}+w_{23}^{3}+w_{31}^{3}\right)+J_{2}^{3} w_{12} w_{23} w_{31}+\frac{J_{1} J_{2} J_{3}}{2}\left[w_{12}^{2}\left(w_{23}+w_{31}\right)+\ldots\right]
\end{aligned}
$$

One can rearrange these terms in terms of the biased two point correlations $\xi_{i j}$, using the leading terms in the expansion, and write the irreducible three point correlation function

$$
\varsigma_{123}=Q\left(\xi_{12} \xi_{23}+\xi_{23} \xi_{31}+\xi_{31} \xi_{12}\right)+Q^{3} \xi_{12} \xi_{23} \xi_{31}+\frac{1}{2} Q\left(T-Q^{2}\right)\left[\xi_{12}^{2}\left(\xi_{23}+\xi_{31}\right)+\ldots\right]
$$

where the terms $Q$ and $T$ are defined by Hermite coefficients of $G(X)$ :

$$
Q=J_{2} / J_{1}^{2} ; T=J_{3} / J_{1}^{3}
$$

The observed irreducible galaxy three point correlation function can be expressed in terms of the 2 point correlations :

$$
\varsigma_{123}(o b s)=Q\left(\xi_{12} \xi_{23}+\xi_{23} \xi_{31}+\xi_{31} \xi_{12}\right)
$$

with $Q$ close to 1 (Groth and Peebles 1977). $\$_{123}$ will thus satisfy the scaling relation to lowest order. The presence of the cubic term $Q^{3} \xi_{1} \xi_{2} \xi_{3}$ is unavoidable, but the mixed term may disappear if $T=Q^{2}$.

The threshold functions in Eq.(7) and (8) are rather unique, having $Q=T=1$, so the mixed term in the three point correlation function disappears, providing the simplest possible 3 point correlation function, called the Kirkwood superposition (Peebles 1980) :

$$
\left\langle G_{1} G_{2} G_{3}\right\rangle=\left(1+\xi_{12}\right)\left(1+\xi_{23}\right)\left(1+\xi_{31}\right)
$$

Not all biasing functions have this simple form of course. For the approximation in BBKS describing the correlations of density peaks one can calculate the expansion coefficients as well, where $G(X)$ is now the density of peaks as a function of the Gaussian height $X$. $\alpha$ and $\beta$ were determined by the properties of the background field.

$$
\begin{aligned}
G(X)=A \exp \left(\alpha X-\frac{1}{2} \beta X^{2}\right) & ; \quad J_{1}=\frac{\alpha}{1+\beta} \\
Q=1-\frac{\beta(1+\beta)}{\alpha^{2}} & ; T=1+\frac{(1+\beta)(2-\beta)}{\alpha^{2}}
\end{aligned}
$$

in agreement with BBKS, except here we also have the detailed amplitudes of the cubic terms, which are present in BBKS in a less obvious way. 


\section{CONCLUSIONS}

Studying general functional forms of Gaussian biasing, we have calculated various higher order correlation functions of the biased density (luminosity) field. We find that these correlations are fully determined by the Hermite coefficients of the nonlinear threshold functions. In the leading order, the amplification of the two point correlations is given by the first coefficient, $J_{1}^{2}$.

For any threshold function the leading term in the 3 point function is $Q\left(\xi_{1} \xi_{2}+\right.$ ...), with $Q=J_{2} / J_{1}^{2}$, always accompanied by a cubic term $Q^{3} \xi_{1} \xi_{2} \xi_{3}$. Generally a mixed cubic term will also be present, but for the special case of sharp clipping this term disappears, and $Q=1$. These arguments can easily be extended to higher order correlation functions, where a similar scaling has been established (Fry and Peebles 1978). If the coefficients $Q, T$ and higher are determined, one could reconstruct the dominant shape of the biasing threshold function $G(X)$.

Observations can potentially restrict the cubic terms in the 3-point function, and therefore provide strong constraints on mechanisms for biasing. However, the cubic terms may only show up in triangles close to equilateral. Clusters of galaxies may be more suitable to look for these effects than galaxies, since they may have preserved the 'initial conditions' more on scales below the correlation length than galaxies. Such work is currently in progress (Bonometto, Lucchin and Mattarrese 1987, Hollosi, Toth and Szalay 1987). Even though the detailed structure of the galaxy 3-point correlation function may be erased by subsequent evolution, the power in the correlation function is still largely due to biasing. A paper by Bonometto, Lucchin and Mattarrese (1987) suggests that even for galaxies there may be traces of such an effect.

If $Q=1$, but there is no cubic term in the 3-point function, we can conclude that Gaussian biasing cannot be responsible for a major amplification of the correlations. No such strong conclusion is possible if the underlying fluctuations were already non-Gaussian, but for sharp clipping the cubic term is present in $\$ 123$, as discussed by Mattarrese, Lucchin and Bonometto (1986).

The author would like to acknowledge useful conversations with B.B. Mandelbrot and M.S.Fall. This research has been supported in Budapest by a grant of the Hungarian Academy of Sciences (OTKA).

\section{REFERENCES}

Bardeen,J.R., Bond,J.R., Kaiser,N. and Szalay,A.S. 1986, Ap.J., 304, 15.

Bonometto,S.A., Lucchin,P. and Mattarrese,S. 1987, Ap.J., in press.

Fry,J.N. 1986, Ap.J., 308, L71.

Fry,J.N. and Peebles,P.J.E. 1978, Ap.J., $221,19$.

Groth,E. and Peebles,P.J.E. 1977, Ap.J., 217,385.

Hollosi,J., Toth,G. and Szalay,A.S. 1987, in preparation.

Jensen,L.G. and Szalay,A.S. 1986, Ap.J., 305, L5.

Kaiser,N. 1984, Ap.J., 284, L9.

Kaiser,N. and Davis,M. 1985, Ap.J., 297, 365.

Mattarrese,S., Lucchin,F. and Bonometto,S.A. 1986, Ap.J., 310, L21.

Peebles,P.J.E. 1980, The Large Scale Structure of the Universe,

(Princeton University Press, Princeton).

Politzer,D. and Wise,M.B. 1984, Ap.J., 285, L1.

Taquu,M.S. 1977, Z. Wahrscheinlichkeitstheorie, 40, 203. 